\title{
La APARICión de la ORQuídea SaCoila lanceolata (ORCHIDOIDEAE: Cranichideae: Spiranthinae) en el ejido de la Ciudad Autónoma de Buenos AIRES
}

\author{
Presence of the orchid Sacoila lanceolata (Orchidoideae: Cranichideae: Spiranthinae) within \\ the limits of the city of Buenos Aires
}

Agustín Sanguinetti ${ }^{1,2,3}$ (D) Hugo Campos ${ }^{4,5}$ (D)

\begin{abstract}
Resumen: A pesar de que la ciudad de Buenos Aires es un territorio antropizado en su totalidad existen reductos de vegetación que albergan plantas nativas de ocurrencia espontánea. Aquí informamos el registro de la orquídea terrestre Sacoila lanceolata (Aubl.) Garay dentro del perímetro de la ciudad. La misma fue hallada en el Arboretum del Parque Sarmiento, una clausura reciente de $1000 \mathrm{~m}^{2}$ donde son conservadas especies nativas de la región ribereña bonaerense. Este registro ocurre en el extremo austral de distribución de la especie, donde las demás citas cercanas se ubican a más de $200 \mathrm{~km}$ de distancia. Dado lo inesperado de este registro discutimos la naturaleza de su aparición y su relevancia como potencial especie bandera.
\end{abstract}

Palabras clave: Chloraea membranacea, especie bandera, Orchidaceae, reserva urbana.

Summary: Even though the city of Buenos Aires is an anthropized territory in its entirety, there are vegetation patches that house spontaneously occurring native plants. We report here the occurrence of the terrestrial orchid Sacoila lanceolata (Aubl.) Garay within the perimeter of the city. It was found in the Arboretum of Parque Sarmiento, a recent enclosure of $1000 \mathrm{~m}^{2}$ where native species of Buenos Aires' riverine region are conserved. This report occurs at the southern limit of the species' distribution, where other nearby records are more than 200 km away. Given the unexpectedness of this event we discuss the nature of its occurrence and its relevance as a potential flag species.

Key words: Chloraea membranacea, flagship species, Orchidaceae, urban reserve.

\section{Introducción}

Orchidaceae, con cerca de 750 géneros (Chase et al., 2015) y 27.000 especies (WCSP, 2021), es una de las familias más numerosas de las plantas vasculares. Posee representantes en todos los tipos de ambientes terrestres, a excepción de desiertos extremos y del continente antártico. La subtribu Spiranthinae, con cerca de 40 géneros y 520 especies (Salazar et al., 2018), es la más diversa en lo que respecta a orquídeas

${ }^{1}$ Departamento de Biodiversidad y Biología Experimental, Facultad de Ciencias Exactas y Naturales, Universidad de Buenos Aires, Pabellón II, Ciudad Universitaria, (C1428EGA) Buenos Aires, Argentina. E-mail: sangos@gmail.com

${ }^{2}$ CONICET-Universidad de Buenos Aires, Instituto de Biodiversidad y Biología Experimental y Aplicada (IBBEA), Pabellón II, Ciudad Universitaria, C1428EGA Buenos Aires, Argentina.

${ }^{3}$ Universidad Nacional de Entre Ríos, Facultad de Bromatología, Pte. Perón 1154, (2820) Gualeguaychú, Entre Ríos, Argentina.

${ }^{4}$ Agrupación Vecinos por la Ecología, Saavedra, Buenos Aires, Argentina.

${ }^{5}$ Universidad CAECE, Licenciatura en Gestión Ambiental, Buenos Aires, Argentina. 
terrestres del neotrópico (Salazar, 2003). La mayoría de las especies de esta subtribu son de hábito terrestre, con raíces carnosas fasciculadas y hojas de posición basal. Las flores se caracterizan en su mayoría por un labelo que abraza a los laterales de la columna en toda su longitud y que posee dos nectarios en la base; por la presencia de un espolón más o menos notable formado en parte por la fusión basal de los sépalos laterales; y por una columna elongada con la antera erecta y el estigma lobulado (Salazar, 2003; Salazar et al., 2018).

Sacoila lanceolata (Aub1.) Garay es una especie de amplia distribución neotropical, con registros desde el norte de México y el estado de Florida en EE. UU. hasta Uruguay y el centro de la Argentina (Guimarães, 2014). Ocupa ambientes secos y abiertos bajo insolación directa a indirecta tal como pastizales o arbustales con suelo arcilloso a pedregoso; también puede ocupar ambientes disturbados (Johnson, 2001). Pueden encontrarse ejemplares de forma aislada o agregados formando conjuntos vistosos. Dentro de Argentina, $S$. lanceolata ocurre en todas las provincias con excepción de las patagónicas, Mendoza y La Pampa. En las provincias de Córdoba y Salta, los registros para esta especie son abundantes, mientras que en las provincias de Buenos Aires y Entre Ríos, y en la República Oriental del Uruguay, son muy escasos (Correa, 1955).

La superficie original de la Ciudad Autónoma de Buenos Aires (CABA) sufrió una profunda y casi total transformación con el cambio de cobertura debido al crecimiento de la urbanización y del territorio construido. Son muy pocos los relictos de vegetación originales remanentes y, aun así, estos se ven afectados con pérdida de diversidad nativa y presencia de especies exóticas (Matteucci et Falcón, 2012). Por otro lado, la modificación del territorio también implica la creación de nuevos ambientes que son colonizados por especies nativas y exóticas generando sucesiones ecológicas complejas (Sirolli et Kalesnik, 2015).

Una especie bandera es, según Samways (citado en Home et al., 2009), aquella que, siendo conocida y carismática, sirve como símbolo o punto focal para elevar la consciencia ambiental. La implementación de políticas y acciones que apunten a la conservación de estas especies pueden colaborar también para la protección del ambiente que habitan. De esta forma, las especies bandera actuarían como "paraguas" aportando a la conservación de las demás especies con las que coexisten. Las orquídeas son un grupo de plantas bandera de alto perfil que concentran numerosos esfuerzos y políticas a nivel local y mundial para su conservación y para la conservación de los ambientes de los que dependen (Gale et al., 2018).

En este trabajo se cita por primera vez a $S$. lanceolata en el distrito de CABA, se discute la naturaleza de este registro, así como la conveniencia de su uso como especie bandera para la manutención y ampliación de las reservas naturales urbanas.

\section{Materiales y Métodos}

El ejemplar de referencia, que se muestra en la Fig. 1, fue fotografiado in situ en el Arboretum "Árboles de Buenos Aires" $\left(34,5520^{\circ} \mathrm{S} ; 58,5002^{\circ} \mathrm{W} ; 15 \mathrm{~m}\right.$ s.n.m.) del Parque Sarmiento de CABA (Fig. 2). De este fueron tomadas 3 flores, disectadas, extendidas y montadas sobre un cartón a modo de exsiccata que fue depositada en el herbario SI del Instituto Darwinion (espécimen Sanguinetti 366, 26-X-2017). El resto del ejemplar fue dejado intacto en el local de modo de permitir su supervivencia.

El Arboretum es una clausura formada en el año 2004 en una franja de $10 \times 100 \mathrm{~m}$ sobre un pastizal nativo con predominancia de las gramíneas Bothriochloa laguroides (DC.) Herter, Nassella neesiana (Trin. \& Rupr.) Barkworth, Piptochaetium bicolor (Vahl) E. Desv. y subarbustos del género Baccharis L. (Fig. 2). Allí se sembraron e implantaron con el correr de los años diversas especies herbáceas y leñosas provenientes de poblaciones de la ribera bonaerense. El Parque Sarmiento se erige sobre lo que fue la chacra de Luis María Saavedra (Piñeiro, 


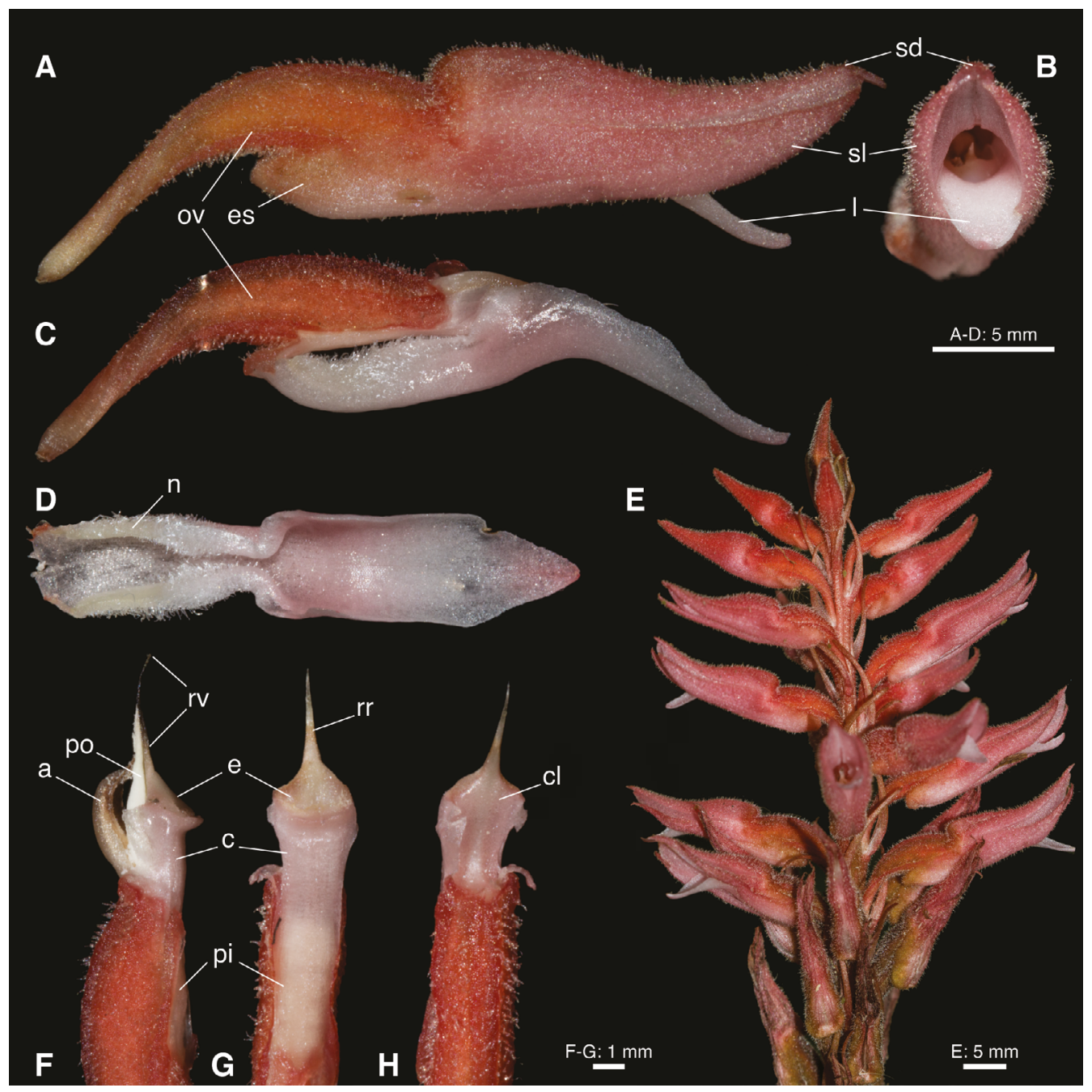

Fig. 1. Ejemplar de Sacoila lanceolata coleccionado en la Ciudad de Buenos Aires (Sanguinetti 366, SI). A: Vista lateral de la flor. B: Vista frontal. C: Vista lateral con sépalos y pétalos removidos. D: Vista adaxial de labelo. E: Inflorescencia. F: Vista lateral de ginostemio. G: Vista ventral de ginostemio con antera y polinario removidos. H: Vista dorsal de ginostemio con antera y polinario removidos. Referencias: a: antera, c: columna, cl: clinandrio, e: estigma, es: espolón, n: nectario, 1: labelo, pi: pie de columna, po: polinario, ov: ovario, rv: rostelo con viscidio, rr: remanente rostelar luego de la remoción del viscidio, sd: sépalo dorsal, sl: sépalo lateral.

Fig. 1. Sacoila lanceolata specimen collected in Buenos Aires City (Sanguinetti 366, SI). A: Flower side view. B: Frontal view. C: Side view without sepals and petals. D: Labellum adaxial view. E: Spike. F: Gynostemium side view. G: Gynostemium ventral view without anther and pollinarium. H: Gynostemium dorsal view without anther and pollinarium. References: a: anther, c: column, cl: clynandrum, e: stygma, es: spur, n: nectary, l: labellum, pi: collumn foot, po: pollinarium, ov: ovary, rv: rostellum with viscidium, rr: rostelar remnant after viscidium removal, sd: dorsal sepal, sl: lateral sepal.

2001) y en la actualidad se encuentra rodeado por una matriz de territorio construido con bajísima conectividad de espacios verdes.

Con el fin de ubicar registros adicionales de zonas cercanas se revisaron herbarios de la región
(BA, BAA, BAF, BBB, LP, LPS, SI; acrónimos según Thiers, 2021) y se consultaron bases de datos en línea (www.inaturalist.com, www. ecoregistros.com, www.floraargentina.edu.ar y Documenta Florae Australis) y bibliografía. 

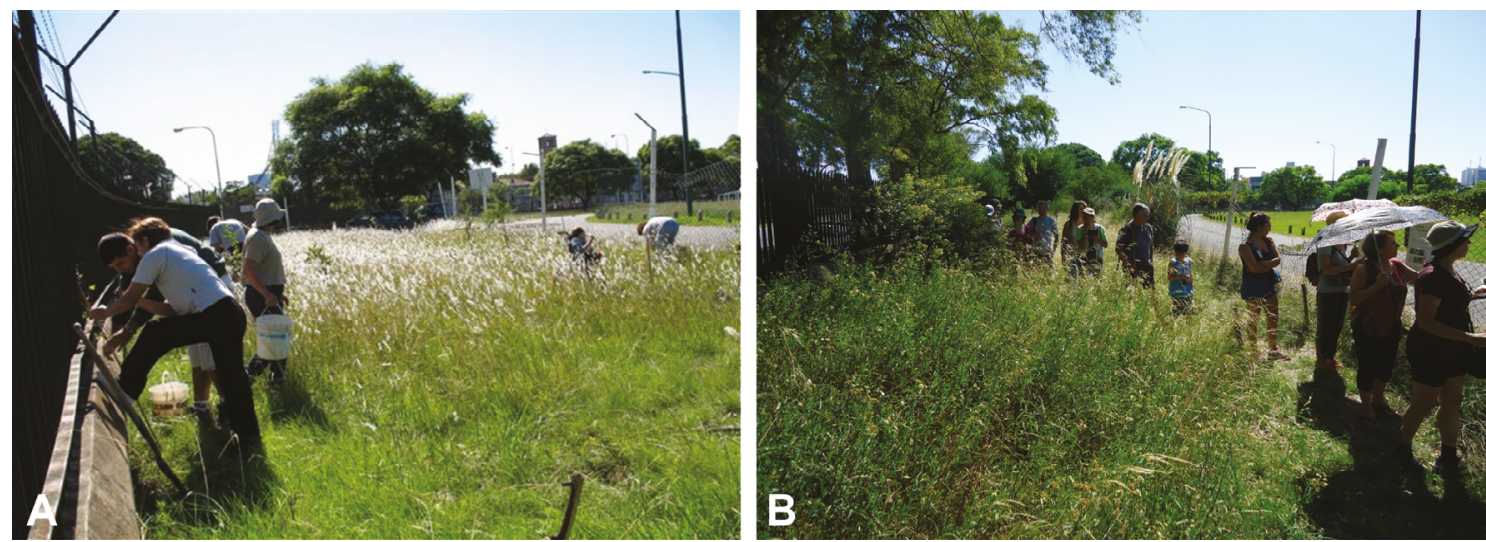

Fig. 2. Paisaje del Arboretum "Árboles de Buenos Aires" del Parque Sarmiento de la Ciudad Autónoma de Buenos Aires. A: Inicio de la clausura del pastizal original en el año 2004. Se destacan las inflorescencias de Botriochloa laguroides. B: Año 2019 donde se puede observar una mayor heterogeneidad de estratos y de biodiversidad.

Fig. 2. Arboretum's "Árboles de Buenos Aires" landscape at Parque Sarmiento, Buenos Aires City. A: Year 2004 when the enclosure began. Botriochloa laguroides` spikes are easily distinguished. B: Year 2019 when more strata and biodiversity heterogeneity is seen.

\section{Resultados}

En estado vegetativo $S$. lanceolata se distingue por sus hojas linear-elípticas, pseudopecioladas (de allí el aspecto lanceolado plasmado en el epíteto) y algo carnosas de 20 a $50 \mathrm{~cm}$ de long. erectas a semierectas que conforman una roseta basal. Cuando florece las hojas marchitan y desarrolla una espiga subpiramidal a cilíndrica algo densa sobre un escapo erecto de hasta $70 \mathrm{~cm}$ (Fig. 1E). Las flores no emiten perfume aparente y son vistosas, de un color rosa pálido a salmón intenso, de hasta $2,5 \mathrm{~cm}$ de longitud y posición horizontal (Fig. 1A, E). Los pétalos están adosados al sépalo dorsal que, en conjunto, cubren a la columna. Los sépalos laterales son erectos y acompañan al labelo, también erecto, que abraza con sus márgenes a la columna. Esto se traduce en una flor de conformación tubular y cerrada con la columna oculta (Fig. 1A, B, C). El labelo posee dos nectarios laterales en su base en forma de callosidades y el néctar secretado se acumula en un breve espolón basal formado por la base fusionada de los dos sépalos y el pie extendido de la columna (Fig. 1A, C, D). La coloración rojiza, la ausencia de fragancia y una conformación tubular con acumulación de copioso néctar en su base son características de un síndrome de polinización ornitófilo. En efecto, las flores son polinizadas por colibríes (Singer et Sazima, 2000). Johnson (2001) y Correa et al. (en prensa) describen extensamente esta especie y Guimarães (2014) provee su lista sinonímica completa.

El ejemplar de $S$. lanceolata fue hallado florecido a mediados de octubre de 2017. Este consistió en una única vara de $50 \mathrm{~cm}$ de altura con una inflorescencia de maduración basípeta con 28 flores de color rosa pálido (Fig. 1E). Algunas de sus flores contaban con el polinario removido, lo que evidenciaba la visita de polinizadores. El ejemplar se encontraba a la media sombra de un "chañar" [Geoffroea decorticans (Gillies ex Hook. \& Arn.) Burkart] y un "coronillo" (Scutia buxifolia Reissek). En las cercanías había ejemplares de "cedrón de monte" [Aloysia gratissima (Gillies \& Hook.) Tronc.], "ñapìnday" [Senegalia bonariensis (Gillies ex Hook. \& Arn.) Seigler \& Ebinger], "camará morado" [Lantana megapotamica (Spreng.) Tronc.] y "malva rosa" (Pavonia hastata Cav.).

La consulta a la base de datos Documenta Florae Australis arrojó que, de un total de 1840 especímenes de orquídeas determinados a nivel de género para la Argentina, 102 (5,54\%) 
corresponden a $S$. lanceolata. Esto confirma que es una especie de aparición frecuente en el país. Ninguno de estos registros corresponde a las provincias de Buenos Aires, Entre Ríos o Santa Fe. En el herbario BAA existe un espécimen proveniente de Junín, Buenos Aires [Colombo s.n., 9-XI-2005 (BAA 25580!)] y en BAF uno del Parque Nacional El Palmar,
Entre Ríos [Cusato \& Rossow 4957, 31-X1992 (BAF 1476!)]. En registros en línea se encontró uno para la periferia de la ciudad de Rosario, Santa Fe (Massi, 2021), y al revisar bibliografía (Pérez et al., 2020) se halló una cita para el departamento de Río Negro, República Oriental del Uruguay [Marchesi \& Vignale s.n., 19-XI-1998 (MVFA 28581)] (Fig. 3).
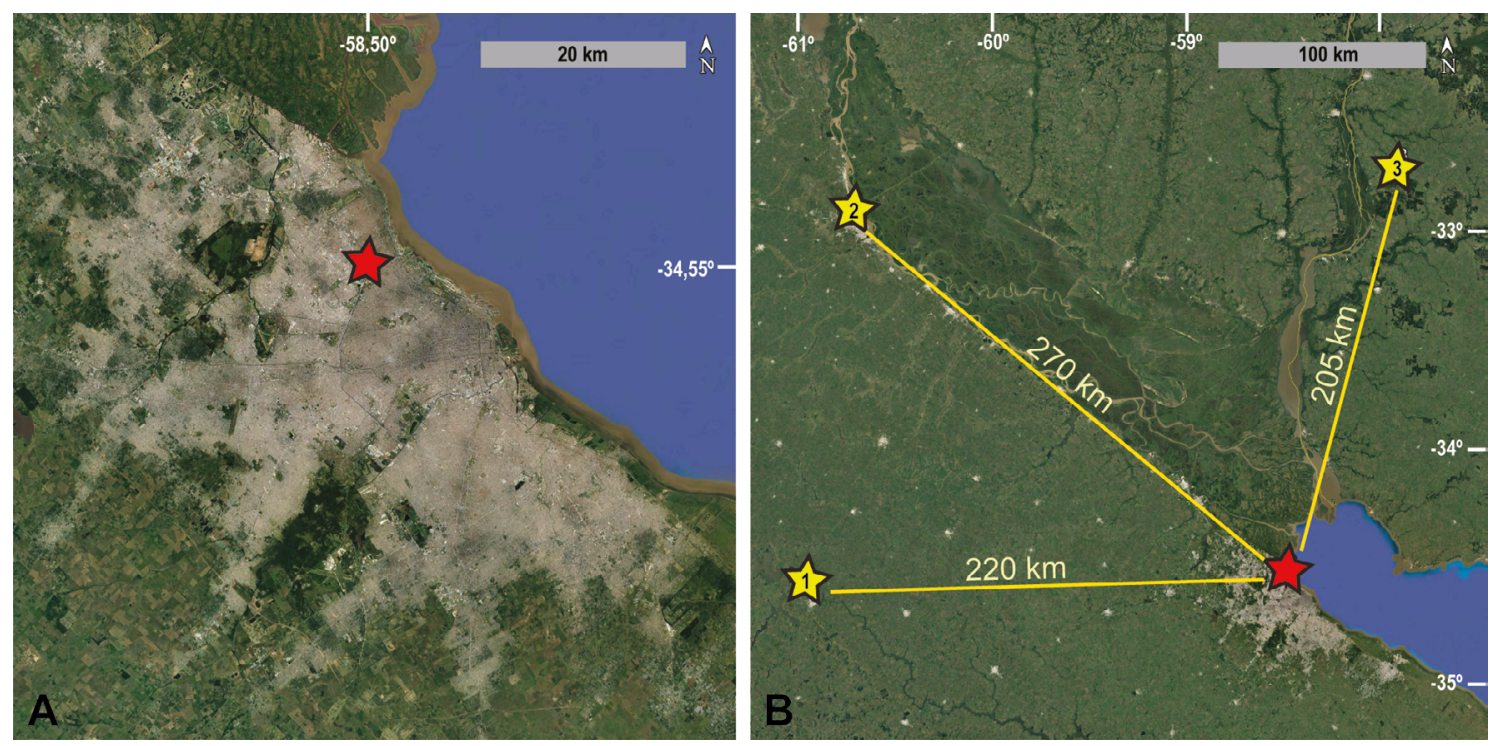

Fig. 3. A: Posición del registro en la matriz urbana de la zona metropolitana de Buenos Aires. B: Relación geográfica con los registros conocidos más próximos (1: Junín, Buenos Aires, Colombo s.n., BAA 25580; 2: Rosario, Santa Fe, Obs. Massi César; 3: Uruguay, Marchesi \& Vignale s.n., MVFA 28581).

Fig. 3. A: Occurrence site within the urban matrix of Buenos Aires metropolitan region. B: Relative position to its closest occurrences (1: Junín, Buenos Aires, Colombo s.n., BAA 25580; 2: Rosario, Santa Fe, Obs. Massi César; 3: Uruguay, Marchesi \& Vignale s.n., MVFA 28581).

\section{Discusión}

El descubrimiento de esta orquídea en el Parque Sarmiento es sorprendente por el hecho de estar ubicado en el núcleo de una matriz totalmente antropizada como lo es el área metropolitana de Buenos Aires. Por otro lado, a pesar de que esta especie es frecuente a nivel nacional, no lo es en la región. Los registros más próximos son muy escasos y se encuentran a más de $200 \mathrm{~km}$ de distancia (Fig. 3). Por esto, surge el interrogante de la naturaleza de la aparición de este único ejemplar y planteamos las siguientes posibilidades:
1) Que sea un ejemplar de una población relictual de la vegetación original de la chacra de Luis María Saavedra. En el parque hay especies nativas arbustivas (e. g., Salvia guaranitica A. St.-Hil. ex Benth.) y herbáceas (ver Materiales y Métodos) que no han sido implantadas y que suponemos que, al menos en parte, corresponden a la vegetación original anterior a la antropización de su alrededor. Análisis de fotografías aéreas muestran que la porción del terreno que corresponde al Arboretum no sufrió movimientos de tierra por lo menos desde 1929 hasta el presente (Ministerio de Desarrollo Urbano 
y Transporte, 2021). A pesar de que fuera del Arboretum el parquizado y el césped se mantienen periódicamente, el hecho de que previamente no haya sido registrada en una zona tan concurrida es llamativo y no refuerza esta hipótesis.

Como contraejemplo de orquídeas que habitaron el perímetro de CABA pero que actualmente no se les conoce ninguna población actual, cabe mencionar el de Habenaria bractescens Lindl. Esta fue recolectada en la ribera original de los barrios de Belgrano [Nuñez s.n., II-1905 (BAF 10311!)] y Recoleta [Spegazzini s.n., III-1880 (BAF 10309!)]. Todo el ambiente costero de CABA y sus bañados fueron profundamente modificados con rellenos que le ganaron superficie al Río de la Plata (Malagnino, 2007) por lo que H. bractescens podría ser considerada una especie extinta para este distrito.

2) Que sea un ejemplar espontáneo surgido de semillas aerotransportadas. Las orquídeas se caracterizan por poseer frutos que producen numerosas semillas, en el orden de miles, muy livianas y que son diseminadas por el viento (Barthlott et al., 2014). Esta es una característica que explica en parte su potencial dispersivo que puede traducirse en una alta tasa de expansión geográfica (Cohen et Ackerman, 2009; Neto et al., 2011), en un alto flujo génico con una consecuente baja estructuración genética espacial (Vanden Broeck et al., 2014; Hedrén et al., 2018), en reiterados eventos de colonización de islas (Hedrén et al., 2018) y hasta en la colonización de espacios antropizados y disturbados (Adamowski, 2006). Esta posibilidad se trataría de un evento extremadamente azaroso de acuerdo con la falta de poblaciones registradas en las cercanías.

3) Que el ejemplar haya sido trasladado como plántula o semilla entre la vegetación bonaerense implantada en el Arboretum. Todos los ejemplares de vegetación que han sido sembrados o trasplantados allí provienen de poblaciones de la ribera bonaerense dentro de un perímetro no mayor a los $60 \mathrm{~km}$. Este caso sería similar al anterior, pero donde el humano es el factor dispersor de los propágulos (Croteau, 2010). Asimismo, también sería un evento muy azaroso dada la ausencia de registros de $S$. lanceolata en zonas ribereñas cercanas.

Recientemente, fue descubierta una pequeña población de la orquídea Serapias parviflora Parl. en la terraza vegetada de un edificio en el centro financiero de Londres, Inglaterra. Esta es la única población de esa especie conocida actualmente en ese país. Los anteriores registros llegan hasta el año 2009 cuando la última población fue destruida (BBC, 2001). Este caso, también sorprendente dado su lugar de aparición, plantea parcialmente el mismo enigma que el de $S$. lanceolata en CABA y puede ser explicado como un ejemplo de semillas aerotrasportadas o plántulas que llegaron junto con el sustrato.

En nuestra opinión, el caso más plausible es el primero, que correspondería a un ejemplar relictual de la vegetación original. Este sería un ejemplo equivalente, en general, al de diversas especies de orquídeas terrestres que persisten en espacios verdes en otras ciudades del mundo (Löki et al., 2019) y, en particular, al de la orquídea Chloraea membranacea Lindl. en la misma ciudad de Buenos Aires.

Las orquídeas son especies carismáticas que llaman la atención del público en general. Chloraea membranacea es una especie nativa común en la región y que posee poblaciones remanentes en algunos espacios verdes de la ciudad (Sanguinetti et al., 2012). Especialmente, C. membranacea se caracteriza por una inflorescencia vistosa de delicadas y estilizadas flores blancas. Estas son algunas de las razones por las que la Reserva Natural Lago Lugano de CABA lleva como isotipo a su flor. Esta especie, a su vez, fue declarada en el año 2017 por la Legislatura de CABA como flor simbólica de la ciudad (Ley 5925).

Las reservas naturales urbanas y periurbanas sirven a fines de esparcimiento, recreativos y de educación. También funcionan como refugios de biodiversidad y pueden formar parte de corredores biológicos para la fauna en tránsito (Haene, 2020). La aparición de $S$. lanceolata en el Arboretum es motivo para que, junto con el ejemplo de C. membranacea, funcionen como especie bandera con el fin de 
incentivar el apoyo público en la manutención y la ampliación de estos espacios verdes urbanos (Home et al., 2009).

\section{Agradecimientos}

A dos revisores anónimos que con sus comentarios colaboraron en mejorar el manuscrito inicial y a los curadores de los herbarios consultados por su asistencia en la búsqueda de especímenes.

\section{Bibliografía}

Adamowski, W. (2006). Expansion of native orchids in anthropogenous habitats. Polish Botanical Studies, 22: $35-44$.

Barthlott, W., Grobe-Veldmann, B. \& Korotkova, N. (2014). Orchid seed diversity: a scanning electron microscopy survey. Englera 32.

BBC. (2021). Rare orchids found in City of London bank's rooftop garden. Disponible: https://www.bbc. com/news/uk-england-london-57439921 (Consulta 10/08/2021).

Chase, M. W., Cameron, K. M., Freudenstein, J. V., Pridgeon A. M., Salazar, G., van den Berg C. \& Schuiteman, A. (2015). An updated classification of Orchidaceae. Botanical Journal of the Linnean Society 177: 151-174. https://doi.org/10.1111/boj.12234

Cohen, I. M. \& Ackerman, J. D. (2009). Oeceoclades maculata, an alien tropical orchid in a Caribbean rainforest. Annals of Botany 104: 557-563. https://doi.org/10.1093/aob/mcn191

Correa, N. M. (1955). Las orquídeas argentinas de la Tribu Polychondreae Schlechter: Subtribu Spiranthinae Pfitzer. Darwiniana 11: 24-88.

Correa, N. M., Múlgura, M. \& Sanguinetti, A. En prensa. Orchidaceae. En Bacigalupo, N., Múlgura, M. \& M. Ponce (eds.), Flora Ilustrada de Entre Ríos (Argentina). Parte I: Pteridófitas, Gimnospermas y Monocotiledóneas (sin las Gramíneas). Instituto de Botánica Darwinion, Buenos Aires.

Croteau, E. K. (2010). Causes and Consequences of Dispersal in Plants and Animals. Nature Education Knowledge 3: 12.

Gale, S. W., Fischer, G. A., Cribb, P. J. \& Fay, M. F. (2018). Orchid conservation: bridging the gap between science and practice. Botanical Journal of the Linnean Society 186: 425-434.

https://doi.org/10.1093/botlinnean/boy003

GuimarÃES, L. R. S. (2014). Filogenia e citotaxonomia do clado Stenorrhynchos (Spiranthinae, Cranichideae, Orchidoideae, Orchidaceae). Tesis doctoral. Instituto de Botânica, São Paulo. 101 pp.

Haene, E. (2020). Biocorredores de la Ciudad Autónoma de Buenos Aires, un modelo demostrativo para la Argentina. Universidad de Belgrano. Buenos Aires, $145 \mathrm{pp}$.

Hedrén, M., Olofsson, S. N. \& Paun, O. (2018). Orchid colonization: multiple parallel dispersal events and mosaic genetic structure in Dactylorhiza majalis ssp. lapponica on the Baltic island of Gotland. Annals of Botany 122: 1019-1032. https://doi.org/10.1093/aob/mcy111

Home, R., Keller, C., Nagel, P., Bauer, N. \& Hunziker, M. (2009). Selection criteria for flagship species by conservation organizations. Environmental Conservation 36: 139-148. https://doi.org/10.1017/S0376892909990051

Johnson, A. E. (2001). Las Orquídeas del Parque Nacional Iguazú. Ed. L.O.L.A., Buenos Aires, Argentina.

Löki, V., Molnár, V. A., Süveges, K., Heimeier, H., Takács, A., Nagy, T., Fekete, R., Lovas-Kiss, Á., Kreutz, K. C. A. J., Sramkó, G. \& Tökölyi, J. (2019). Predictors of conservation value of Turkish cemeteries: A case study using orchids. Landscape and Urban Planning 186: 36-44.

https://doi.org/10.1016/j.landurbplan.2019.02.016

Massi, C. (2021). Registro de Sacoila lanceolata. Disponible: https://www.inaturalist.org/ observations/45556785 (Consulta 10/08/2021).

Matteucci, S. D. \& Falcón, M. (2012). Efectos de la urbanización sobre la biodiversidad. En ATHOR, J. (Ed.), Buenos Aires, la historia de su paisaje natural, pp. 272-291. Fundación de Historia Natural Félix de Azara y Universidad Maimónides. Buenos Aires.

Ministerio de Desarrollo Urbano y Transporte. Gobierno de la Ciudad de Buenos Aires. (2021). Fotografías Aéreas. Disponible: http:// ssplan.buenosaires.gob.ar/webfiles/mapa_aereas2 (Consulta 10/08/2021).

Malagnino, E. (2007). Geomorfología. En Nabel, P. \& D. Kullock (eds.), Atlas Ambiental de Buenos Aires. Museo Argentino de Ciencias Naturales, CONICET y Gobierno de la Ciudad de Buenos Aires.

Neto, L. M., Miranda, M. R. \& Cruz, D. (2011). Zeuxine strateumatica (Orchidaceae) goes south: a first record for Brazil. Kew Bulletin 66: 155-158. https://doi.org/10.1007/s12225-011-9271-2 
Pérez, L. M., Pessoa, E. M. \& Alves, M. (2020). Lista comentada de Orchidaceae en Uruguay y su distribución en ambientes y eco-regiones. Lankesteriana 20: 359-395. https://doi.org/10.15517/lank.v20i3.45193

Piñeiro, A. G. (2001). El Museo Saavedra en sus primeros 80 años. Historias de la ciudad, Año 3, Nro 12.

Salazar, G. A. (2003). Spiranthinae. En Pridgeon, A. M., CribB, P. J., Chase, M. W. \& F. N. Rasmussen (eds.), Genera Orchidacearum vol. 3 Orchidoideae part 2, Vanilloideae, pp. 164-278. Oxford University Press, New York.

Salazar, G. A., Batista, J. A. N., Cabrera, L. I., van Den Berg, C., Whitten, W. M., Smidt, E. C., Buzatto, C. R., Singer, R. B., Gerlach, G., JiménezMachorro, R., Radins, J. A., Insaurralde, I. S., Guimarães, L. R. S., de Barros, F., Tobar, F., Linares, J. L., Mújica, E., Dressler, R. L., Blanco, M. A., Hágsater, E. \& Chase, M. W. (2018). Phylogenetic systematics of subtribe Spiranthinae (Orchidaceae: Orchidoideae: Cranichideae) based on nuclear and plastid DNA sequences of a nearly complete generic sample. Botanical Journal of the Linnean Society 186: 273-303. https://doi.org/10.1093/botlinnean/box096

Sanguinetti, A., Buzatto, C. R., Pedron, M., Davies, K. L., Ferreira, P. M. D. A., Maldonado, S. \& Singer, R. B. (2012). Floral features, pollination biology and breeding system of Chloraea membranacea Lindl. (Orchidaceae: Chloraeinae). Annals of Botany 110: 1607-1621. https://doi.org/10.1093/aob/mcs221

Singer, R. B. \& SAZima, M. (2000). The pollination of Stenorrhynchos lanceolatus (Aublet) L. C. Rich. (Orchidaceae: Spiranthinae) by hummingbirds in southeastern Brazil. Plant Systematics and Evolution 223: 221-227. https://doi.org/10.1007/ BF00985281

Sirolli, H. \& Kalesnik, F. A. (2015). Composición, estructura y tendencia sucesional de un bosque de aliso de río (Tessaria integrifolia), en la Reserva Ecológica Costanera Sur, Ciudad Autónoma de Buenos Aires, Argentina. Historia Natural, Tercera Serie, 5: 109-124.

ThiERs, B. (2021). Index Herbariorum: a global directory of public herbaria and associated staff. New York Botanical Garden's Virtual Herbarium, http:// sweetgum.nybg.org/ih

Vanden Broeck, A., Van Landuyt, W., Cox, K., De Bruyn, L., Gyselings, R., Oostermeijer, G., Valentin, B., Bozic, G., Dolinar, B., Illyés, Z. \& MergeaY, J. (2014). High levels of effective longdistance dispersal may blur ecotypic divergence in a rare terrestrial orchid. BMC Ecology, 14: 20. https://doi.org/10.1186/1472-6785-14-20

WCSP. (2021). World Checklist of Selected Plant Families. Royal Botanic Gardens, Kew. Disponible: http://wcsp.science.kew.org (Consulta 10/08/2021). 